\title{
Clay mineral reactivity in the critical zone: exploring emergent coupled processes using the reactive transport code CrunchClay
}

\author{
CHRISTOPHE TOURNASSAT ${ }^{1,2}$ AND CARL STEEFEL ${ }^{2}$ \\ ${ }^{1}$ University of Orléans \\ ${ }^{2}$ Lawrence Berkeley National Laboratory \\ Presenting Author: christophe.tournassat@univ-orleans.fr
}

Clay minerals are ubiquitous on Earth surface. They are major components in many soil types, and are the main components of extended sedimentary stratigraphic layers. Illite and smectite alone may constitute $30 \mathrm{w} \%$ of all sedimentary rocks. The nanoto micrometer size of clay minerals particles, combined with their layered structures, provide them with high specific surface area values, among the highest known for natural materials, and are responsible for their high adsorption capacities for a range of chemicals spanning from natural organic matter to emerging organic and inorganic pollutants. Clay mineral adsorption reactivity is indeed recognized to have a major influence on nutrient availability, carbon preservation, and contaminant mitigation in soils. In the last decades, a growing number of studies also evidenced the redox (reversible) reactivity of Fe(II) and $\mathrm{Fe}(\mathrm{III})$ present in clay layers, which could have an influence on elements cycles and contaminant degradation and/or mobility. The accuracy of predictions of geochemical interactions in many surficial environments is thus partly dependent on our capability to consider the various aspects of clay mineral reactivity in our modeling approaches. Clay mineral layers are also negatively charged as a result of heterovalent substitutions of cations in their structure. This property is responsible for their well-known cation exchange capacity, but it is also responsible for specific physical properties influencing the transfer of chemical species in the porosity of clayey media in a non-classical (Darcian and Fickian) way. The prediction of nutrient and contaminant mass transfers in natural clayey environments is thus made very difficult because of a strong coupling of a complex chemical reactivity with none less complex transport properties. In this presentation, we will show how newly developed reactive transport modeling capabilities in the code CrunchClay could help overcoming these difficulties. 